\title{
Cybervictimización y cyberagresión en estudiantes universitarios: problemas emocionales y uso problemático de nuevas tecnologías
}

\author{
Santiago Resett ${ }^{1}$ y Paula Romina Putallaz ${ }^{2}$
}

\begin{abstract}
RESUMEN
La cybervictimización y la cybeagresión constituyen importantes factores de riesgo para la salud mental de los individuos ya que, por ejemplo, en algunos casos la cybervictimización puede conducir al suicidio de las víctimas. Si bien en la actualidad en la Argentina se ha prestado una notable atención a estas problemáticas, pocos estudios se han llevado a cabo en dicho país para determinar los correlatos psicosociales de la cybervictimización y la cyberagresión en estudiantes universitarios. Se constituyó una muestra intencional de 410 alumnos universitarios que cursaban estudios regulares de primero a quinto año en universidades privadas de Paraná, Entre Ríos, Argentina. El 75\% eran mujeres. Las edades iban de 17 a 36 años, con una media de 21.8 años. El 57\% vivía con ambos padres. Los participantes contestaron un cuestionario de cyberbullying, escalas sobre depresión y ansiedad-estrés y un cuestionario de uso problemático de nuevas tecnologías. La participación voluntaria, el anonimato y la confidencialidad fueron asegurados. Los resultados indicaban que un $31 \%$ era cybervictimizado y un $27 \%$ era cyberagresor, que más mujeres que varones eran cybervíctimas y que no existian diferencias a este respecto en cyberagresión. Las cybervíctimas tenían puntajes más altos en depresión, ansiedad-estrés y uso problemático de nuevas tecnologías, mientras los cyberagresores solo mostraban puntajes más altos de uso problemático de nuevas tecnologías. En la discusión se analizan las implicancias de estos hallazgos, se proveen las limitaciones de la investigación y se brindan sugerencias para futuros estudios.
\end{abstract}

Palabras clave: cybervictimización, cyberagresión, problemas emocionales, nuevas tecnologias, universitarios.

\section{Cybervictimization and cyberaggression in college students: internalizing problems and problematic use of new technology}

\begin{abstract}
Cybervictimization and cyberbullying is an important risk factor for the mental health of individuals, for example, suffering cybervictimization can lead to suicide in victims. However, few studies have been conducted in Argentina to determine their psychological correlates in college students. Therefore, an intentional sample of 410 university students who studied regular first to fifth year in private universities of Paraná, Entre Ríos, Argentina was constituted; $75 \%$ were women. The ages ranged from 17 years to 36, with an average of 21.8 years. The participants completed Calvete et al. Cyberbullying Questionnaire, Lovibond and Lovibond Depression, Anxiety and Stress Scale, and Labrador et al. Problematic Use of New Technology Questionnaire. A 27\% was cybervictim and a $31 \%$ was cyberaggressor, more women than men were cybervictims, and no differences were detected regarding cyberaggression. The results showed that cybervictims presented more depression, anxiety-stress and problematic use of new
\end{abstract}

\footnotetext{
${ }_{1}^{1}$ Universidad Católica Argentina, Consejo Nacional de Investigaciones Científicas y Técnicas (CONICET), Argentina; resettsantiago@gmail.com

2 Universidad de Palermo, Argentina.
} 
technology, whereas cyberaggressor only showed more problematic use of new technology. In the discussion we analyze the implications of these findings and we provide limitations and suggestions for future studies.

Keywords: cybervictimization, cyberbullying, internalizing problems, new technology, college student.

El bullying o acoso escolar es un importante factor de riesgo para numerosos problemas de ajuste psicosocial (Card \& Hodges, 2008; Card, Isaacs, \& Hodges, 2007; Troop-Gordon, 2017). El bullying se define como agresión intencional, repetida y con un desbalance de fuerza física o mental entre la víctima y el agresor (Card \& Hodges, 2008). Así, numerosos estudios se han llevado a cabo tanto en el primer mundo (naciones de América del Norte y norte de Europa), como en los países en vías de desarrollo, para determinar los niveles de victimización. Estos estudios trasnacionales indicaron que la problemática estaba extendida en distintos países y no se limitaba a una región en particular (e.g., Bradshaw, Crous, Rees, \& Turner, 2017; Cook, Williams, Guerra, \& Kim, 2009).

En los últimos años el desarrollo de las nuevas tecnologías, como su popularidad entre los más jóvenes, dio lugar al cyberbullying (Mason, 2008; Olweus \& Limber, 2018). A pesar de que todavía se debate acerca de cómo medirlo y definirlo (Mehari, Farrel, \& Le, 2014; Patchin \& Hinduja, 2015) -el primer estudio a este respecto es de hace una década atrás (Mason, 2008)-, la mayoría de los investigadores señala que es una agresión intencional y repetida que ocurre a través de celulares, computadoras u otros medios electrónicos (Patchin \& Hinduja, 2015; Sontang, Clemans, Graber, \& Lyndon, 2011), y de la cual la víctima no puede defenderse fácilmente (Slonje \& Smith, 2008; Smith, del Barrio, \& Tokunawa, 2013). Este tipo de acoso presenta características diferentes y se diferencia del bullying por ser más anónimo, rápido, cómodo, se produce en cualquier momento y se extiende más allá del ámbito escolar (Li, 2006), por lo cual para muchos autores podría ser más negativo para la salud mental y tener una mayor incidencia que el bullying (Sticca \& Perren, 2013). De esta manera, los investigadores han presentado visiones diferentes entre sí acerca del cyberbullying (Kowalski \& Limber, 2013). Algunos sugieren que el cyberbullying es meramente una extensión del bullying realizado a través de nuevas tecnologías (Hinduja \& Patchin, 2008; Olweus, 2012). Otros autores, en cambio, sugirieron que el cyberbullying difiere del bullying en algunos aspectos importantes (por ejemplo, anonimato, audiencia masiva, entre otros) y también indicaron que -aunque comparten algunas características comunes- son diferentes cualitativamente y con correlatos socioemocionales diferentes al bullying (Kowalski \& Limber, 2013).

$\mathrm{Si}$ bien el bullying ha sido investigado extensamente en niños $\mathrm{y}$ adolescentes del primer mundo y en los países en vías de desarrollo, el cyberbullying se ha estudiado menos. Del mismo modo, el cyberbullying ha sido todavía menos estudiado en muestras de estudiantes universitarios, a pesar del gran uso de las nuevas tecnologías que hacen estos alumnos, del incremento de los hechos de cyberbullying y de sus nefastas consecuencias para las víctimas (Schenk \& Fremouw, 2012; Waasdorp \& Bradshaw, 2015). Incluso en los países del primer mundo -donde se genera la mayoría de la investigación en psicología del desarrollo (Facio, Resett, Mistrorigo, \& Micocci, 
2006)- la investigación empírica a este respecto es escasa (Asher, Stark, \& Fireman, 2017).

En lo relativo a los pocos estudios internacionales disponibles en alumnos universitarios, Kowalski, Giumetti, Schroeder y Reese (2012) hallaron que un $30 \%$ de los estudiantes universitarios habian sufrido cyberbullying. Schenk y Fremouw (2012) encontraron que un 9\% era víctima de cyberbullying. Como se ve, existe mucha variabilidad en los porcentajes. Sin embargo, los pocos estudios empíricos en estudiantes universitarios sugieren una variación de entre 10\%-35\% en la involucración en el cyberbullying, cifras que -incluso- pueden llegar a ser mayores que las detectadas en muestras de adolescentes (Kokkinos, Antoniadou, \& Markos, 2014; Schenk, Fremouw, \& Keelan, 2013).

Con respecto a la cybervictimización, esta se ha asociado a una gran cantidad de problemas psicológicos, tales como ansiedad, depresión -como uno de sus correlatos psicológicos con un mayor tamaño del efecto-, y, en algunos casos, suicidio (Mehari et al., 2014). Los problemas emocionales o internalizantes son un relevante factor de riesgo para una apropiada transición hacia la adultez (La Greca \& Harrison, 2005), como puede ser el caso de los alumnos universitarios. Por otra parte, la depresión y la ansiedad son algunos de los más frecuentes trastornos mentales a la cybervictimización (Hughes \& Gullone, 2010). En lo relativo a los correlatos de problemas emocionales de ser cybervictimizado, Schenk y Fremouw (2012) encontraron que las víctimas sufrian de mayor depresión, ansiedad, fobia social y paranoia. Algunas investigaciones en muestras de alumnos universitarios detectaron que ser cybervictimizado se asociaba con mayor depresión y estrés (Bauman \& Baldasare, 2015; Tennant, Demaray, Cyle, \& Malecki, 2015). Así, se sabe mucho de los correlatos psicosociales de la cybervictimización, pero menos se han estudiado los correlatos psicosociales de los perpetradores de la cyberagresión (Slonje, Smith, \& Frisén, 2013). En lo relativo a los estudios disponibles a este respecto, los cyberagresores no presentan mayores problemas de salud mental, con la única excepción de una mayor conducta antisocial (Kowalski \& Limber, 2013). Tampoco mostraban altos niveles de problemas emocionales, sino que -al contrario- ostentaban una imagen positiva de sí mismo, como baja sintomatología depresiva y ansiosa y altos niveles de autoestima (Resett \& Gámez-Guadix, 2017). Sin embargo, investigaciones han hallado que la cyberagresión se asociaba con un peor funcionamiento psicosocial (Wong, Chan \& Cheng, 2014). No obstante, la mayoría de estos estudios relativos a la cyberagresión son con muestras de adolescentes. Sería interesante evaluar qué sucede con la cyberagresión y sus correlatos emocionales en una etapa de la vida como la adultez emergente (Arnett, 2000) -en la cual la agresión no es vista tan positivamente- y en un ambiente como el universitario.

Otro importante correlato de la cybervictimización y la cyberagresión es el uso problemático de nuevas tecnologías (Gámez-Guadix, Orue, Smith, \& Calvete, 2013). Este se define como una preocupación constante por las nuevas tecnologías, incapacidad para controlar su uso (necesidad de estar conectado todo el tiempo a internet) y continuar con su uso compulsivo, a pesar de los problemas para la salud fisica y mental (Young, 2011). Algunos estudios detectaron que el uso problemático de nuevas tecnologias se asociaba con mayor cybervictimización y cyberbullying (Gámez-Guadix, Borrajo, \& Almendro, 2016). 
En los países latinoamericanos, la investigación sobre el cyberbullying es prácticamente inexistente. Con respecto a las escasas investigaciones disponibles, un estudio en Colombia -aunque en lo referido al bullying- halló un $10 \%$ de víctimas en alumnos universitarios (Hoyos de los Ríos, Romero Santiago, Valega Mackenzie, \& Molinares Brito, 2009). Otro estudio en Colombia detectó que casi un $27 \%$ de alumnos universitarios cyberagredía a sus pares (Redondo, Luzardo, \& Rangel, 2016).

Por todo lo dicho y debido a la escasa literatura científica, resulta de gran importancia evaluar los niveles de cybervictimización y cyberagresión en alumnos universitarios de la Argentina y sus correlatos de problemas emocionales y de uso problemático de nuevas tecnologías. De este modo, el objetivo del presente estudio fue determinar si los alumnos universitarios cybervictimizados y cyberagresores difieren en el nivel de problemas emocionales (depresión, ansiedad y estrés) y uso problemático de nuevas tecnologias, en comparación con los grupos no involucrados.

Hipótesis: Los alumnos universitarios cybevictimizados presentan mayores niveles de problemas emocionales y mayor uso problemático de nuevas tecnologias, en comparación con los no involucrados. Mientras que los cyberagresores presentan mayor uso problemático de nuevas tecnologías, pero no difieren en su nivel de problemas emocionales.

\section{METODOLOGÍA}

\section{Tipo de estudio}

Se trató de un estudio descriptivo ex post-facto de corte transversal con una metodología tipo encuesta.

\section{Participantes}

Se constituyó una muestra intencional no probabilística de 410 alumnos universitarios que cursaban estudios de psicologia, psicopedagogía y abogacía en universidades privadas de Paraná, Entre Ríos, Argentina. Los criterios de inclusión eran: ser mujer o varón, tener entre 17 a 36 años, ser alumno regular, cursar entre primero a quinto año de estudios de psicología, psicopedagogía o abogacía en universidades privadas de Paraná, Entre Ríos. E1 75\% eran mujeres y el resto varones. Las edades iban de 17 a 36 años, con una media de $21.8(D E=4.8)$. El $30 \%$ cursaba primer año, el $18 \%$, segundo, $10 \%$, tercero, $24 \%$ cuarto y $18 \%$, quinto año. El $57 \%$ vivía con su madre y su padre y el $18 \%$ trabajaba.

\section{Instrumentos}

Los participantes completaron un cuestionario sociodemográfico: sexo, edad, año que cursaban, universidad y carrera que cursaban.

El Cuestionario de Cyberbullying de Calvete, Orue, Estévez, Villardón y Padilla (2010). Como el fin era medir cybervictimización y cyberagresión, se empleó dicho cuestionario. Se compone de dos escalas diferentes, una para medir cyberagresión y una para medir cybervictimización. El cuestionario consta de 14 preguntas sobre cyberagredir y 14 sobre cybervictimización. Un ejemplo de pregunta de cyberagresión es "Colgué enlaces donde aparecían rumores, chismes etc. de un compañero para que las leyeran otras personas". Un ejemplo de pregunta para cybervictimización es: "Me enviaron mensajes 
amenazantes o insultantes con la Internet o el celular". Las preguntas de cyberagresión inquieren sobre: enviar mensajes amenazantes o insultantes; colgar o enviar imágenes amenazantes o insultantes; poner enlaces o imágenes insultantes o desagradables; escribir o difundir chismes; colgar enlaces donde aparecen chismes o rumores; conseguir la contraseña y enviar mensajes para hacer quedar mal a alguien; grabar o tomar fotos mientras se burlan de alguien; mandarlas a través de las redes sociales; grabar o tomar fotos mientras le pegan a alguien; mandarlas a través de las redes sociales; difundir secretos a través de las redes sociales; excluir de las redes sociales; grabar o tomar fotos de comportamiento sexual y mandarlas a través de las redes sociales. La subescala de cybervictimización pregunta la frecuencia con la que los adolescentes han sufrido dichos comportamientos como víctimas. El formato de respuesta utilizado para evaluar la frecuencia de cada comportamiento es una escala tipo Likert con las siguientes alternativas 0 (Nunca), 1 (1 o 2 veces), 2 (3 o 4 veces) y 3 (5 o más veces). Sus propiedades están bien establecidas en muestras españolas y mexicanas, las cuales demostraron una estructura bifactorial y $\alpha$ de Cronbach .90 y .79 para la escala de cyberagresión y cybervictimización, respectivamente (Calvete et al., 2010; Gámez-Guadix, Villa-George, \& Calvete, 2014). También en la Argentina sus bondades psicométricas fueron detectadas. En lo referente a su validez de constructo, en nuestro país se comprobó la asociación de las dos escalas con la depresión, la ansiedad y los rasgos de la personalidad. Asimismo, se detectaron consistencias internas adecuadas (Resett \& Gámez-Guadix, 2017). En esta investigación las $\alpha$ de Cronbach fueron .72. y .73, respectivamente.

Escala de Depresión, Ansiedad y Estrés (DASS-21) de Lovibond y Lovibond (1995). Se aplicó para medir los problemas emocionales. Se trata de una medida de autoinforme conformada por subescalas que miden tres estados emocionales negativos: depresión, ansiedad y estrés. La subescala Depresión evalúa disforia, desesperanza, desinterés por la vida, anhedonia, desaprobación de sí mismo y desmotivación. La subescala Ansiedad mide la activación del sistema nervioso autónomo y la experiencia subjetiva del afecto ansiedad. La escala de Estrés evalúa el nivel de excitación crónica no especifica: nerviosismo, dificultad para relajarse, irritabilidad e hiperreactividad ante los estímulos. Un ejemplo de un item de depresión es: "Me parecía que no tenía nada por qué vivir", un ejemplo de ansiedad era: "Tenía la boca seca" y un ejemplo de estrés era "Reaccionaba exageradamente ante ciertas situaciones". En el DASS-21, una versión reducida del DASS, cada subescala consta de siete preguntas en lugar de los 14 items de la versión original. Como al sujeto se le pide que describa cómo se sintió durante los últimos siete días, el DASS-21 es una medición de estado más que de rasgo. Los items constan de cuatro niveles de severidad/frecuencia que van desde 0 (no me pasaba) hasta 3 (me pasaba muchísimo). Los puntajes más altos indican mayor nivel de emocionalidad negativa. En cuanto a sus propiedades psicométricas, Lovibond y Lovibond (1995) informaron unos $\alpha$ de Cronbach de .81 para Depresión, .73 para Ansiedad y .81 para Estrés. Presenta, además, evidencia de validez factorial concurrente (por ejemplo, con las escalas Beck de Depresión y Ansiedad) y discriminativa. Sus propiedades están bien establecidas en la Argentina con una adecuada estructura factorial, con $\alpha$ de Cronbach $.88, .89$ y .89, respectivamente, y validez de constructo de las subescalas con el Inventario de Depresión Beck y la Escala de Síntomas Psicosomáticos de Rosenberg para medir ansiedad (Facio, Micocci, \& Resett, 2008). La alta correlación entre ansiedad y estrés encontrada en muestras 
argentinas conlleva a que las mismas se sumen en un mismo puntaje (Facio et al., 2008). En la presente investigación el $\alpha$ de Cronbach fue .87 para depresión y .89 para ansiedad-estrés, respectivamente.

Cuestionario de uso problemático de nuevas tecnologías de Labrador, Villadangos, Crespo y Becoña (2013). Como se deseaba evaluar el uso problemático de nuevas tecnologías, se empleó este instrumento. Este es un cuestionario autoadministrado de 26 preguntas dirigido a identificar el riesgo de conductas problemáticas en el uso de Internet, redes sociales, computadoras videojuegos, celular y televisión. Se compone de tres subescalas: frecuencia de uso (6 preguntas), frecuencia de su uso problemático (6 preguntas) y problemas asociados con el uso de las tecnologías mencionadas (7 preguntas). Un ejemplo de pregunta del cuestionario es: "¿Te ponés mal cuando por algún motivo no podés usar las nuevas tecnologias?". Todas las preguntas presentan cuatro opciones de respuestas que se puntúan de 1 a 4 . Las opciones de respuesta para la frecuencia de uso van de 1 (nunca) a 4 (todos los días) y para las otras dos subescalas van de 1 (nunca) a 4 (siempre). Su estructura factorial y validez convergente están ampliamente comprobadas en muestras españolas y presentó $\alpha$ de Cronbach por encima de .70 para las tres subescalas (Labrador et al., 2013). Sus propiedades psicométricas fueron detectadas también en nuestro país y demostró evidencia de validez de constructo, como lo era su asociación con el trolling, y adecuadas $\alpha$ de Cronbach (Gonzalez Caino \& Resett, 2018). En la presente investigación, el $\alpha$ de Cronbach para frecuencia de uso problemático fueron .82 y .83 , respectivamente. En el presente estudio se trabajó con la escala de frecuencia de uso problemático de nuevas tecnologías y problemas asociados con su uso. No se informará sobre la subescala uso de nuevas tecnologías, ya que son objeto de otra investigación.

\section{Procedimiento}

Se aseguró a los jóvenes la confidencialidad, el anonimato de las respuestas y la participación voluntaria. Las encuestas se aplicaron en el horario normal de clases o en las horas libres de los estudiantes.

\section{Análisis de datos}

Los datos se analizaron en el Programa Estadístico para las Ciencias Sociales SPSS (por su acrónimo en inglés) versión 22. Se extrajeron estadísticos descriptivos (medias, porcentajes, entre otros), como inferenciales para determinar si existían diferencias de sexo y si las cybervíctimas, cyberagresores y grupos no involucrados diferian en las variables dependientes ( $\chi^{2}$ y MANOVAs análisis multivariados de la varianza). Los porcentajes de estudiantes cybervictimizados, cyberagresores y no involucrados se conformaron a partir de dicotomizar las 14 preguntas de cybervictimización y cyberagresión $(0=$ no involucrado, $1=$ cybervíctima, $0=$ no involucrado y 1 = cyberagresor). Se usó un criterio conservador de dos o tres veces al mes como mínimo para dicotomizar las preguntas de acuerdo con estudios previos (Kowaski \& Limber, 2013; Olweus, 2012). Por ejemplo, si un estudiante contestaba que era victimizado en algunas de las 14 preguntas de cybervictimización, al menos dos o tres veces al menos, se consideraba como cybervíctima. Lo mismo sucedía para cyberagresión. 


\section{RESULTADOS}

Antes de comenzar con el objetivo específico del presente trabajo, se presentarán los porcentajes de estudiantes cybervictimizados y no involucrados y cyberagresores y no involucrados discriminados por sexo. En la tabla 1 se muestran los resultados de cybervíctimas y no involucrados, según sexo, mientras que en la tabla 2 se presentan los de cyberagresores y no involucrados, según sexo. Los resultados indicaron que un $31 \%$ era cybervíctima y $27 \%$ era cyberagresor, respectivamente. Se llevó a cabo una prueba de $\chi^{2}$ para determinar si el sexo introducía diferencias en ser cybervíctima y ser cyberagresor, respectivamente. Los resultados indicaron que existian diferencias de sexo marginales en cybervíctimas, pero no existían diferencias en cyberagresores $\chi^{2}(1,409)=3.48, p<.07$ y $\chi^{2}(1,409)=1.21, p<$ .27 , respectivamente, como se muestra en las tablas 1 y 2 . Como se percibe en la tabla 1, más varones (79\%) eran no involucrados, en comparación con las mujeres (67\%); en cambio, más mujeres (33\%) que varones $(21 \%)$ eran cybervíctimas. Los residuales corregidos arrojaban 1.9 -valores muy cercanos al 1.96, esto es, celdas en las que existen más casos de lo que debería haber-.

Tabla 1.

No involucrados y cybervíctimas según sexo.

\begin{tabular}{|c|c|c|c|}
\hline Grupo & Varón & Mujer & Totales \\
\hline No involucrados & $79 \%(n=46)$ & $67 \%(n=236)$ & $69 \%(n=282)$ \\
\hline Cybervíctimas & $21 \%(n=12)$ & $33 \%(n=116)$ & $31 \%(n=128)$ \\
\hline Totales & $100 \%(n=58)$ & $100 \%(n=352)$ & $100 \%(n=410)$ \\
\hline
\end{tabular}

Tabla 2.

No involucrados y cyberagresores según sexo.

\begin{tabular}{|c|c|c|c|}
\hline Grupo & Varón & Mujer & Totales \\
\hline No involucrados & $67 \%(n=39)$ & $74 \%(n=261)$ & $69 \%(n=300)$ \\
\hline Cyberagresores & $33 \%(n=19)$ & $26 \%(n=91)$ & $31 \%(n=110)$ \\
\hline Totales & $100 \%(n=58)$ & $100 \%(n=352)$ & $100 \%(n=410)$ \\
\hline
\end{tabular}

En la tabla 3 se muestran las medias y desvíos típicos en la escala de cybervictimización y cyberagresión.

Tabla 3.

Medias y desvíos típicos en cybervictimización

y cyberagresión $(n=410)$.

\begin{tabular}{lc|}
\hline Variable & $\boldsymbol{M}(\boldsymbol{D E})$ \\
\hline Cybervictimización & $1.21(2.06)$ \\
Cyberagresión & $1.24(2.36)$ \\
\hline
\end{tabular}

Con respecto al objetivo específico de este trabajo -determinar si los alumnos cybervíctimas y cyberagresores diferian en los niveles de problemas emocionales y en el uso problemático de nuevas tecnologías (frecuencia de uso problemático y problemas asociados con el uso)-, se decidió llevar a cabo una serie de MANOVAs. Primeramente, se ejecutó una prueba de Levene para determinar la homogeneidad de las varianzas para las cuatro variables dependientes entre los grupos cybervictimizados y no involucrados. Los resultados indicaron varianzas no homogéneas para depresión y ansiedad- 
estrés $p \mathrm{~s}<.001 \mathrm{y}$ homogéneas para frecuencia de uso problemático y problemas asociados con el uso $p \mathrm{~s}>.145$. De este modo, se procedió a realizar el MANOVAs, pero informando un estadístico más robusto para desigualdad en las varianzas como es $T$ de Pillai, el cual también era apropiado al tener cada grupo una $N$ de más de 30 casos (282 de no involucrados versus 128 de cybervíctimas), como indican numerosos metodólogos (Allen \& Bennett, 2008; Tabachnick \& Fidell, 2007). Al llevar a cabo el MANOVAs con los puntajes en problemas emocionales (depresión y ansiedad-estrés) y uso problemático de nuevas tecnologias (frecuencia de uso problemático y problemas asociados con el uso) como variables dependientes, y la pertenencia al grupo de cybervictimización versus no involucrados como factor entre sujetos, se hallaron diferencias significativas $T$ de Pillai $=.15, F(4,406)=17.84, p<.001$, $\eta^{2}=15 \%$. Las diferencias, según la pertenencia a los grupos, existían en las cuatro variables dependientes -ver tabla 4-.

Tabla 4.

Medias, desvíos típicos y estadísticos univariados en problemas emocionales y uso problemático de nuevas tecnologías según cybervictimización.

\begin{tabular}{l|ccc|}
\hline $\begin{array}{l}\text { Variable } \\
\text { dependiente }\end{array}$ & $\begin{array}{c}\text { No involucrado } \\
(\boldsymbol{n}=\mathbf{2 8 2}) \\
\boldsymbol{M}(\boldsymbol{D E})\end{array}$ & $\begin{array}{c}\text { Cybevíctima } \\
(\boldsymbol{n}=\mathbf{1 2 8}) \\
\boldsymbol{M ( D E )}\end{array}$ & Estadísticos univariados \\
\hline Depresión & $1.41(0.46)$ & $1.71(0.65)$ & $F(1)=28.11, \eta^{2}=6 \%$ *** \\
Ansiedad-estrés & $1.39(0.38)$ & $1.76(0.53)$ & $F(1)=61.90, \eta^{2}=13 \% \%^{* * *}$ \\
Frecuencia de uso problemático & $8.38(3.42)$ & $9.67(3.74)$ & $F(1)=11.76, \eta^{2}=3 \%^{* * *}$ \\
Problemas asociados con el uso & $11.47(3.45)$ & $13.17(3.80)$ & $F(1)=19.69, \eta^{2}=5 \% \%^{* * *}$ \\
\hline Nota: ${ }^{* * *} p<.001$ & & & \\
\hline
\end{tabular}

Con respecto a la cyberagresión, en primer lugar se llevó a cabo un contraste de Levene para determinar la homogeneidad de las varianzas de las variables dependientes entre los grupos cyberagresores y no involucrados. Los resultados indicaban valores no significativos para las cuatro variables dependientes $p s>.08$, por lo cual se asumían varianzas homogéneas. Al llevar a cabo un MANOVAs con los puntajes en problemas emocionales (depresión y ansiedad-estrés) y uso problemático de nuevas tecnologías (frecuencia de uso problemático y problemas asociados con el uso) como variables dependientes y la pertenencia al grupo de cyberagresión versus no involucrados como factor entre sujetos, se hallaron diferencias significativas según la pertenencia a los grupos $\lambda$ de Wilks $=.90, F(4,406)=11.32, p<.001, \eta^{2}=10 \%$. Las diferencias existian en frecuencia de uso problemático y problemas asociados con el uso ver tabla 5-.

Tabla 5.

Medias, desvíos típicos y estadísticos univariados en problemas emocionales y uso problemático de nuevas tecnologías según cyberagresión.

\begin{tabular}{|c|c|c|c|}
\hline $\begin{array}{l}\text { Variable } \\
\text { dependiente }\end{array}$ & $\begin{array}{c}\text { No involucrado } \\
(n=300) \\
M(D E)\end{array}$ & $\begin{array}{c}\text { Cyberagresor } \\
(n=110) \\
M(D E)\end{array}$ & Estadísticos univariados \\
\hline Depresión & $1.48(0.57)$ & $1.56(0.50)$ & $F(1)=1.56, \eta^{2}=0 \%$ \\
\hline Ansiedad-estrés & $1.49(0.48)$ & $1.55(0.42)$ & $F(1)=1.18, \eta^{2}=0 \%$ \\
\hline Frecuencia de uso problemático & $8.20(3.17)$ & $10.37(4.09)$ & $F(1)=31.62, \eta^{2}=7 \%^{* * *}$ \\
\hline Problemas asociados con el uso & $11.35(3.32)$ & $13.79(3.90)$ & $F(1)=39.15, \eta^{2}=9 \%$ *** \\
\hline
\end{tabular}




\section{DISCUSIÓN}

El propósito del presente trabajo era determinar si los alumnos universitarios cybervictimizados y cyberagresores diferian en sus correlatos de problemas emocionales y de uso problemático de nuevas tecnologías, en comparación con los no involucrados, ya que -a pesar de la relevancia de la problemática- en las naciones latinoamericanas el tema casi no ha sido investigado. Así se constituyó una muestra de 410 estudiantes universitarios que realizaban estudios en universidades privadas de Paraná, Entre Ríos, Argentina. Los participantes contestaron el Cuestionario de Cyberbullying de Calvete et al. (2010), el DASS-21 para depresión y ansiedad-estrés de Lovibond y Lovibond (1995) y el Cuestionario de uso problemático de nuevas tecnologias de Labrador et al. (2013).

Se halló un $31 \%$ de alumnos cybervictimizados y un $27 \%$ de cyberagresores. Si bien no hay datos nacionales para comparar los hallazgos, estudios internacionales, como los de Kowalski et al. (2012), también detectaron un porcentaje similar con un 30\% de cybervíctimas. También un estudio en Colombia halló alrededor de un $27 \%$ de cyberagresores (Redondo et al., 2016). Estos hallazgos también ponen de manifiesto que el cyberbullying también está presente en el nivel universitario. Como sugieren muchos estudios (Kokkinos et al., 2014; Schenk et al., 2013), no solo este comportamiento está presente en el nivel universitario, sino que -en ocasiones- su prevalencia es mayor que el encontrado en muestras de adolescentes. Los altos niveles de cyberbullying hallados en el ambiente universitario pueden explicarse por la relación docente-alumno menos personalizada de dicho nivel -en comparación con la de la escuela secundaria o primaria- y por un ambiente menos estructurado que el de los niveles educativos anteriores. En la universidad, por otra parte, los jóvenes no son supervisados en el uso de redes sociales y uso del celular, por lo que esto podría ser un factor de riesgo para un mayor cyberbullying (Walrave \& Heirman, 2011). Además, hay que tener presente que la población de estudiantes de los primeros cursos -a los que se puede considerar adultos emergentes (Arnett, 2000) - están hoy en día más cerca de ser adolescentes que de ser adultos, por lo cual las formas de relacionarse están íntimamente ligados al uso de redes sociales y celulares (Dobarro González, Tuero Herrero, Bernardo Gutiérrez, Herrero Diez, \& Álvarez-García, 2018).

Si bien no era el objetivo de este trabajo, se halló que más mujeres que varones eran cybervictimizadas, mientras que el sexo no introducía diferencias en la cyberagresión. Como el cyberbullying es una agresión indirecta, esto podría llevar a concluir que las mujeres podrian ser tanto más victimizadas que agresoras (Kowalski et al, 2014). A pesar de que algunos estudios apoyan esta hipótesis (e.g., Kowalski \& Limber, 2007), otras investigaciones no han encontrado diferencias entre varones y mujeres a este respecto (Hinduja \& Patchin, 2008; Slonje \& Smith, 2008). No obstante, otras han detectado que los hombres son más agresores que las mujeres (Li, 2006); al mismo tiempo, algunos trabajos hallaron que los varones agreden más, mientras las mujeres son más victimizadas (Sourander et al., 2010). Sin embargo, la mayoría de las investigaciones evaluaron muestras adolescentes. También las inconsistencias a este respecto pueden deberse a las distintas muestras, los instrumentos de evaluación y los rápidos cambios en las nuevas tecnologias. Muchos estudios hipotetizan que las diferencias más grandes a este respecto se dan en el bullying físico y no en el cyberbullying, en el cual la fuerza fisica no cuenta 
(del Barrio, Martín, Montero, Gutiérrez, Barrios, \& De Dios, 2008). Por otra parte, es posible que las diferencias de sexo emerjan de acuerdo con el dispositivo empleado (celulares, computadoras o redes sociales) para sufrir la cybervictimización o para llevar a cabo la cyberagresión.

En lo referente a los correlatos de problemas emocionales, se observó que las cybervíctimas presentaban mayor depresión y ansiedad-estrés, mientras que los cyberagresores no presentan mayores puntajes en dichas variables, en comparación con los no involucrados. Estos resultados concuerdan con numerosos estudios internacionales sobre la asociación entre la cybervictimización y los problemas emocionales (Kowalski et al., 2014; Mehari et al., 2014). Algunas investigaciones en estudiantes universitarios detectaron que sufrir de cyberbullying predecía la sintomatología depresiva, controlando los niveles de bullying (Tennant et al., 2015).

En lo relativo a los cyberagresores, las investigaciones indicaron que ellos no presentaban problemas de este tipo y que su salud mental era similar a la de los no involucrados (Resett \& Gamez-Guadix, 2017). Sin embargo, otras investigaciones detectaron que los cyberagresores sí mostraban mayores problemas emocionales (Bonanno \& Hymel, 2013; Patchin \& Hinduja, 2010; Schenk et., 2013).

Se observó que tanto los estudiantes cybervictimizados como los cyberagresores presentaban un mayor uso problemático de nuevas tecnologías -en comparación con los no involucrados-, como usarlas en exceso o tener problemas asociados a su uso, o no poder controlar el tiempo que se les dedicaba. Esto es concordante con muchos estudios extranjeros que demostraron que usarlas en exceso o sin supervisión adulta se asociaba con el cyberbullying (Gámez-Guadix et al., 2013; Gámez-Guadix et al., 2016). Cabe aclarar que la mayoría de los efectos detectados eran de tamaño mediano entre 3\% y 8\%-, con la excepción de ansiedad-estrés para cybervictimización (13\%) y problemas asociados con el uso para cyberagresión (9\%), los cuales eran de tamaño grande. Sin embargo, se necesitan investigaciones longitudinales para determinar la direccionalidad de la causalidad entre el uso de nuevas tecnologías y el cyberbullying. ¿Es el uso problemático del celular, por ejemplo, una causa o una consecuencia de la cyberagresión/cybervictimización? Esto es, el pasar mucho tiempo con este dispositivo y con su uso patológico ¿conlleva a cyberagredir? o, por el contrario, una pauta de problemas de conducta o externalizantes -entre ellas la agresión- ¿conlleva también a un mal uso de las nuevas tecnologías? Por otra parte, se puede hipotetizar que una tercera variable no evaluada aquí por ejemplo, una personalidad con rasgos de alto neuroticismo y baja agradabilidad- es la que hace covariar al cybebullying y al uso problemático de nuevas tecnologías.

En la actualidad se debate si el cyberbullying es una mera extensión del bullying u otro fenómeno cualitativamente diferente (Kowalski \& Limber, 2013; Kubiszewski, Fontaine, Potard, \& Auzoult, 2015). Del mismo modo, también se discute si sufrir de cyberbullying o de bullying es más negativo para la salud mental (Sticca \& Perren, 2013). Los resultados del presente estudio indicaban que sufrir de cyberbullying -incluso en alumnos universitarios- se asociaba con correlatos psicosociales negativos, como mayores problemas emocionales. Se comprobó también que la cybervictimización en dicha etapa de la vida puede conducir a graves consecuencias (Schwartz, 2010), como el suicidio. Futuros estudios deberian examinar si hay un mayor nivel de bullying o 
cyberbullying en dichas poblaciones y cuál de los dos es más negativo para la salud mental. Muchos estudios se preguntaron si los efectos de la cybevictmización en los alumnos universitarios son o no normativos (Asher et al., 2017). Si bien en el presente estudios se hallaron niveles no menores de cyberbullying (un $31 \%$ de cybervíctimas y un $27 \%$ de cyberagresores), los resultados del presente trabajo no son generalizables por tratarse de una muestra de conveniencia.

Este estudio tiene una serie de limitaciones. Primero, el haber sido llevado a cabo con una muestra intencional no probabilística de Paraná, Entre Ríos, lo cual impide su generalización a la población de estudiantes universitarios. Además, el tamaño de la muestra era de 410 alumnos, por lo cual se trataba de una muestra con limitado poder estadístico. Por otra parte, la muestra era desproporcionada con respecto al sexo, ya que contaba con mayor cantidad de mujers que varones. Segundo, el diseño transversal no permite inferir la direccionalidad de la causalidad: puede ser que la cybervictimización afecte a la salud mental, como también que el tener una peor salud mental vuelva a los estudiantes universitarios un blanco más vulnerable para el cyberbullying. Tampoco este tipo de estudio permite evaluar cómo va evolucionando el fenómeno a través del tiempo. Tercero, el haber usado soel autoinforme como único instrumento de recolección de datos es una limitación (se sabe que dicha técnica conlleva al sesgo subjetivo y la falta de honestidad en las respuestas). Asimismo, el haber medido todas las variables con la misma técnica de recolección de datos aumenta artificialmente las relaciones entre las variables.

Sin embargo, el presente trabajo pone de manifiesto que el cyberbullying está también presente en el ámbito universitario y que se asociaba a una peor salud mental de los sujetos. Futuros estudios deberian examinar esta problemática en muestras aleatorias, de mayor tamaño y de diversas ciudades de la Argentina. Sería deseable que midieran la problemática longitudinalmente para observar cómo va evolucionando el fenómeno a través del tiempo, lo cual también permitiría evaluar la direccionalidad de la causalidad. Asimismo, investigaciones longitudinales deberian examinar la continuidad entre ser acosado en la escuela secundaria y en el ámbito universitario. ¿Continúan las victimas del cyberbullying en la escuela secundaria siendo victimizados en la universidad? Por otra parte, se debería evaluar el bullying y el cyberbullying para observar cuál es un mejor predictor de una peor salud mental. Finalmente, se deberian usar otras técnicas complementarias para recolectar los datos, como nominaciones de pares, para superar las conocidas limitaciones del autoinforme.

\section{REFERENCIAS}

Allen, P. \& Bennett, K. (2008). SPSS for the Health and Behavioural Sciences. South Melbourne, Australia: Thomson.

Arnett, J. J. (2000). Emerging adulthood: A theory of development from the late teens through the twenties. American Psychologist, 55, 469-480. https://doi.org/10.1037/0003-066X.55.5.469

Asher, Y, Stark, A., \& Fireman, G. D. (2017). Comparing electronic and traditional bullying in embarrassment and exclusion scenarios. Computers in Human Behavior, 76, 2634. https://doi.org/10.1016/j.chb.2017.06.037

Bauman, S. \& Baldasare, A. (2015). Cyber aggression among college students: Demographic differences, predictors of distress, and the role of the university. Journal of College Student Development, 56(4), 317-330. https://doi.org/10.1353/csd.2015.0039

Bonanno, R. A. \& Hymel, S. (2013). Cyber bullying and internalizing difficulties: Above and beyond the impact of traditional forms of bullying. Journal of Youth and Adolescence, 42, 685-697. https://doi.org/10.1007/s10964-013-9937-1 
Bradshaw, J. R., Rees, G., Crous, G., \& Turner, N. (2017). Comparing children's experiences of schoolsbased bullying across countries. Children and Youth Services Review, 80, 1-10. https://doi.org/10.1016/j.childyouth.2017.06.060

Calvete, E., Orue, I., Estévez, A., Villardón, L., \& Padilla, P. (2010). Cyberbullying in adolescents: Modalities and aggressors' profile. Computers in Human Behavior, 26, 1128-1135. https://doi.org/10.1016/j.chb.2010.03.017

Card, N. A. \& Hodges, E. V. (2008) Peer victimization among school children: correlations, causes, consequences, and considerations in assessment and intervention. School Psychology Quarterly, 23, 451-461. https://doi.org/10.1037/a0012769

Card, N. A., Isaacs, J., \& Hodges, E. (2007). Correlates of school victimization: Recommendations for prevention and intervention. En J. E. Zins, M. J. Elias, \& C. A. Maher (Eds.), Bullying, victimization, and peer harassment: A handbook of prevention and intervention (pp. 339-366). Nueva York: Haworth Press.

Cook, C. R., Williams, K. R., Guerra, N., \& Kim, T. (2009). Variability in the prevalence of bullying and victimization: A cross-national and methodological analysis. En S. Jimerson, S. Swearer, \& D. Espelage (Eds.), The International Handbook of School Bullying (pp. 347-362). Mahwah: Erlbaum.

del Barrio, C., Martín, E., Montero, I., Gutiérrez, H., Barrio. A., \& De Dios, M. (2008). Bullying and social exclusion in Spanish secondary schools: National trends from 1999 to 2006. International Journal of Clinical and Health Psychology, 8(3), 657-677.

Dobarro González, A., Tuero Herrero, E., Bernardo Gutiérrez, A., Herrero Díez, F., \& Álvarez-García, D. (2018). Un estudio innovador sobre acoso on-line en estudiantes universitarios. Revista d'Innovació Docent Universitària, 10, 131-142.

Facio, A., Micocci, F., \& Resett, S. (2008). Haciéndose adulto: relaciones interpersonales y problemas emocionales y de conducta en la adultez emergente. Proyecto de Investigación de la Universidad Nacional de Entre Ríos (UNER). Paraná: UNER.

Facio, A., Resett, S., Mistrorigo, C., \& Micocci, F. (2006). Los adolescentes argentinos. Cómo piensan y sienten. Buenos Aires: Editorial Lugar.

Gámez-Guadix, M., Borrajo, E., \& Almendros, C. (2016). Risky online behaviors among adolescents: longitudinal relations among problematic internet use, cyberbullying perpetration, and meeting $\begin{array}{llll}\text { strangers online. Journal of } & \text { Behavioral 100-107. }\end{array}$ https://doi.org/ 10.1556/2006.5.2016.013

Gámez-Guadix, M., Orue, I., Smith, P. K., \& Calvete, E. (2013). Longitudinal and reciprocal relations of cyberbullying with depression, substance use and problematic Internet use among $\begin{array}{lrrr}\text { adolescents. Journal of } & \text { Adolescent } & \text { Health, } & \text { 53, 446-452. }\end{array}$ https://doi.org/10.1016/j.jadohealth.2013.03.030

Gámez-Guadix, M., Villa-George, F., \& Calvete, E. (2014). Psychometric properties of the Cyberbullying Questionnaire (CBQ) among Mexican Adolescents. Violence and Victims, 29, 232247. https://doi.org/10.1891/0886-6708.VV-D-12-00163R1

Gonzalez Caino, P. \& Resett, S. (2018, mayo). Predicting trolling behavior from dark personality, problematic use of new technologies, and sex. Trabajo aceptado para ser presentado en la Conferencia Anual de las Ciencias Psicológicas, San Francisco, Estados Unidos.

Hinduja, S. \& Patchin, J. W. (2008). Cyberbullying: An exploratory analysis of factors related to offending and victimization. Deviant Behavior, 29, 129-156. https://doi.org/10.1080/01639620701457816

Hoyos de los Ríos, O. L., Romero Santiago, L., Valega Mackenzie, S., \& Molinares Brito, C. (2009). El maltrato entre iguales por abuso de poder y exclusión social en estudiantes de una universidad privada de la ciudad de Barranquilla. Pensamiento psicológico, 6(13), 109-125.

Hughes, E. K. \& Gullone, E. (2010). Reciprocal relationships between parent and adolescent internalizing symptoms. Journal of Family Psychology, 24(2), 115-124. https://doi.org/10.1037/a0018788

Kokkinos, C.M., Antoniadou, N., \& Markos, A. (2014) Cyber-bullying: An investigation of the psychological profile of university student participants. Journal of Applied Developmental Psychology, 35(3), 204-214. https://doi.org/10.1016/j.appdev.2014.04.001

Kowalski, R. M. \& Limber, S. P. (2007). Electronic bullying among middle school students. Journal of Adolescent Health, 41(6, Suppl.), 22-30. https://doi.org/10.1016/j.jadohealth.2007.08.017

Kowalski, R. M. \& Limber, S. P. (2013). Psychological, physical, and academic correlates of cyberbullying and traditional bullying. Journal of Adolescent Health, 53, 13-20. https: / / doi.org/10.1016/j.jadohealth.2012.09.018

Kowalski, R. M., Giumetti, G. W., Schroeder, A. N., \& Lattanner, M. R. (2014). Bullying in the digital age: A critical review and meta-analysis of cyberbullying research among youth. Psychological Bulletin, 14O(4), 1073-1137. https://doi.org/10.1037/a0035618

Kowalski, R. M., Giumetti, G. W., Schroeder, A. N., \& Reese, H. H. (2012). Chapter 14 Cyber bullying among college students: Evidence from multiple domains of college life. En L. A. Wankel \& C. Wankel (Eds.), Misbehavior online in higher education (pp. 293-321). Nueva York: Emerald Group Publishing Limited.

Kubiszewski, V., Fontaine, R., Potard, C., \& Auzoult, L. (2015). Does cyberbullying overlap with school bullying when taking modality of involvement into account? Computers in Human Behavior, 43, 49-57. doi: $10.1016 /$ j. chb.2014.10.049

La Greca, A. M. \& Harrison, H. W. (2005). Adolescent peer relations, friendships and romantic relationships: Do they predict social anxiety and depression? Journal of Clinical Child and Adolescent Psychology, 34, 49-61. https://doi.org/10.1207/s15374424jccp3401_5

Labrador, F. J., Villadangos, S. M., Crespo, M., \& Becona, E. (2013). Desarrollo y validación del cuestionario de uso problemático de nuevas tecnologias (UPNT). Anales de psicología, 29(3), 836-847.

Li, Q. (2006). Cyberbullying in Schools, A research of gender differences. School Psychology International, 21, 
157-170. https://doi.org/10.1177/0143034306064547

Lovibond, S. \& Lovibond, P. (1995). Manual for the Depression Anxiety Stress Scales. Sydney: The Psychology Foundation of Australia.

Mason, K. L. (2008). Cyberbullying: A preliminary assessment for school personnel. Psychology in the School, 45(4), 323-348. https://doi.org/10.1002/pits.20301

Mehari, K. R., Farrell, A. D., \& Le, A. H. (2014). Cyberbullying among adolescents: Measures in search of a construct. Psychology of Violence, 4(4), 399-415. https://doi.org/10.1037/a0037521

Olweus, D. (2012). Invited expert discussion paper: Cyberbullying: An overrated phenomenon? European Journal of Developmental Psychology, 9(5), 520- 538. https://doi.org/10.1080/17405629.2012.682358

Olweus, D. \& Limber, S. (2018). Some problems with cyberbullying research. Current Opinion in Psychology, 19, 139-143. https://doi.org/10.1016/j.copsyc.2017.04.012

Patchin, J. W. \& Hinduja, S. (2010). Cyberbullying and self-esteem. Journal of School Health, 80(12), 614621. https://doi.org/10.1111/j.1746-1561.2010.00548.x

Patchin, J. W. \& Hinduja, S. (2015). Measuring cyberbullying: Implications for research. Aggression and Violent Behaviour, 23, 69-74. https://doi.org/10.1016/j.avb.2015.05.013

Redondo, J., Luzardo, M., \& Rangel, K. J. (2016) Conducta agresiva en una muestra de estudiantes de tres colegios de la ciudad de Bucaramanga, Colombia. Revista Encuentros, 14(1), 31-40. https://doi.org/10.15665/re.v14i1.667

Resett, S. \& Gámez-Guadix, M. (2017). Traditional bullying and cyberbullying: Differences in emotional problems, and personality. Are cyberbullies more Machiavellians? Journal of Adolescence, 61, 113-116. https://doi.org/10.1016/j.adolescence.2017.09.013

Schenk, A. M. \& Fremouw, W. J. (2012). Prevalence, psychological impact, and coping of cyberbully victims among college students. Journal of $\quad$ School $\quad$ Violence, 11(1), 21-37. https: / / doi.org/ 10.1080/15388220.2011.630310

Schenk, A. M., Fremouw, W. J., \& Keelan, C. M. (2013). Characteristics of college cyberbullies. Computers in Human Behavior, 29(6), 2320-2327. https://doi.org/10.1016/j.chb.2013.05.013

Schwartz, J. (2010). Bullying, suicide, punishment (Vol. 2). New York Times.

Slonje, R. \& Smith, P. K. (2008). Cyberbullying: A new type of bullying? Scandinavian Journal of Psychology, 49, 147-154. https://doi.org/10.1111/j.1467-9450.2007.00611.x

Slonje, R., Smith, P. K., \& Frisén, A. (2013). The nature of cyberbullying, and strategies for prevention. Computers in Human Behavior, 29(1), 26-32. https://doi.org/10.1016/j.chb.2012.05.024

Smith, P., del Barrio, C., \& Tokunaga, R. (2012). Definitions of bullying and cyberbullying: How useful are the terms? En S. Bauman, J. Walker, \& D. Cross (Eds.), Principles of cyberbullying research. definitions, measures and methodology (pp. 26-40). Nueva York y Londres: Routledge.

Sontag, L. M., Clemans, K., Graber, J. A., \& Lyndon, S. T. (2011). Traditional and cyber aggressors and victims: A comparison of psychosocial characteristics. Journal of Youth and Adolescence, 40, 392- 404. https: / / doi.org/10.1007/s 10964-010-9575-9

Sourander, A., Klomek, A. B., Ikonen, M., Lindroos, J., Luntamo, T., Koskelainen, M.,... Henenius, H. (2010). Psychosocial risk factors associated with cyberbullying among adolescents. Archives of General Psychiatry, 67, 720-728. https://doi.org/10.1001/archgenpsychiatry.2010.79

Sticca, F. \& Perren, S. (2013). Is cyberbullying worse than traditional bullying? Examining the differential roles of medium, publicity, and anonymity for the perceived severity of bullying. Journal of Youth and Adolescence, 42(5), 739-50. https://doi.org/10.1007/s10964-012-9867-3

Tabachnick, B. G. \& Fidell, L. S. (2007). Using multivariate statistics. Boston: Pearson/Allyn \& Bacon.

Tennant, J. E., Demaray, M. K., Coyle, S., \& Malecki, C. K. (2015). The dangers of the web: Cybervictimization, depression, and social support in college students. Computers in Human Behavior, 50, 348-357. https://doi.org/10.1016/j.chb.2015.04.014

Troop-Gordon, W. (2015). Peer victimization in adolescence: The nature, progression, and consequences of being bullied within a developmental context. Journal of Adolescence, 55, 116-128. https://doi.org/10.1016/j.adolescence.2016.12.012

Waasdorp, T. E. \& Bradshaw, C. P. (2015). The overlap between cyberbullying and traditional bullying. Journal of Adolescent Health, 56(5), 483-488. https:/ doi.org/ 10.1016/j.jadohealth.2014.12.002

Walrave, M. \& Heirman, W. (2011) Cyberbullying: Predicting victimization and perpetration. Children \& Society, 25(1), 59-72. https://doi.org/10.1111/j.1099-0860.2009.00260.x

Wong, D. S. W., Chan, H. C., \& Cheng, C. H. K. (2014). Cyberbullying perpetration and victimiza- tionamong adolescents in Hong Kong. Child Youth Service Review, 36, 133-140. https://doi.org/10.1016/j.childyouth.2013.11.006

Young, K. S. (2011). Clinical assessment of Internet-addicted clients. En K. S. Young \& C. N. de Abreu (Eds.), Internet addiction: A handbook and guide to evaluation and treatment (pp. 19-34). Hoboken, Nueva Jersey: John Wiley \& Sons.

Recibido 07-03-2018 | Aceptado 06-06-2018

Este trabajo se encuentra bajo una Licencia Creative Commons Atribución 4.0 Internacional que permite a terceros utilizar lo publicado siempre que se dé el crédito pertinente a los autores y a Psicodebate 